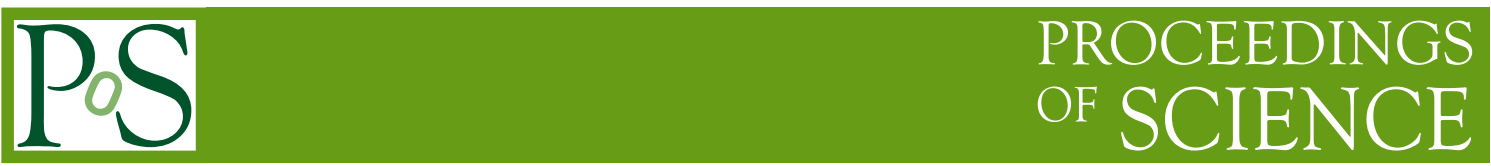

\title{
Potential for New Phenomena in Dijet Events with the ATLAS Detector at $\sqrt{s}=13 \mathrm{TeV}$
}

\section{Jeff DANDOY* on behalf of the ATLAS Collaboration}

University of Chicago (US)

E-mail: jdandoy@uchicago.edu

\begin{abstract}
With the start of Run II of the Large Hadron Collider, pp collisions at the center of mass energy of $13 \mathrm{TeV}$ will open a great potential for observing physics beyond the Standard Model. New physics beyond the Standard Model could couple to the strong force, making searches in jet events extremely exciting. The increase in center of mass energy greatly enhances the reach of searches using dijet events. Shortly after the start of data taking, the sensitivity to new heavy resonant particles will surpass the limits set with the full Run I dataset. The ability to observe non-resonant deviations from the SM such as structure within quarks themselves is also greatly enhanced from the new operating conditions of the LHC. The discovery potential of new physics is presented for both resonant and non-resonant new physics in dijet events.
\end{abstract}

XXVII International Symposium on Lepton Photon Interactions at High Energies

17-22 August 2015

Ljubljana, Slovenia

${ }^{*}$ Speaker. 


\section{Motivation}

As the Large Hadron Collider (LHC) enters a new energy frontier at $\sqrt{s}=13 \mathrm{TeV}$ a natural place to look for beyond the Standard Model (BSM) physics is in the dijet channel. Many BSM models predict new particles which interact through the strong force and may dominantly decay into jets. The cross section for many BSM models may also rise significantly with increases in center of mass energy, requiring only a relatively small amount of luminosity before deviations from the Standard Model (SM) can be seen. New physics could take the form of resonances from phenomena such as quantum black holes $(\mathrm{QBH})$ to previously unseen quark excitations $\left(q^{*}\right)^{1}$. Nonresonant discrepancies with the Standard Model could also arise through new interactions beyond our energy scale $\Lambda$, such as contact interactions.

Previous searches with the ATLAS detector [1] at $\sqrt{s}=7-8 \mathrm{TeV}$ did not reveal any deviations from the SM. The strategies used by these analyses remain valid and can quickly be updated for the new energy frontier. The dijet resonance search [2] looks for any unexpected bumps in the smooth quantum chromodynamics (QCD) dijet mass $\left(m_{j j}\right)$ spectrum. The dijet angular search [3] looks for any unexpected features in the opening angles of the dijet system. The SM predicts the QCD background to be dominated by t-channel processes, leading to more forward jets (more parallel to the beamline) than central jets (transverse to the beamline). Any excess of dijet events in the central region of the detector could point to BSM physics.

\section{Analysis}

The dijet resonance and angular analyses focus on two distinct observables: $m_{j j}$ vs. $\chi=$ $e^{\left|y_{1}-y_{2}\right| 2}$. Yet they are also complementary as both observables can be sensitive to the same models, and any new physics found in one analysis can be cross checked in the other. Both share much of the same phase space of dijet events, requiring unprescaled single jet triggers with an efficiency selection on the leading jet of $p_{T}>410 \mathrm{GeV}$. The most central events are selected in the resonance analysis, with $y^{*}=\frac{y_{1}-y_{2}}{2}<0.6$. This selection is relaxed to 1.7 for the angular analysis to allow for QCD dominant control regions at large $\chi$. In the angular analysis $m_{j j}$ is required to be greater than $2.5 \mathrm{TeV}$, while this is relaxed to $1.1 \mathrm{TeV}$ for the resonance analysis to populate QCD only control regions at low $m_{j j}$.

The resonance analysis uses a data-driven estimation of the dominant QCD background. The data is fit with a traditional function that is insensitive to narrow resonances:

$$
f(x)=p_{1}(1-x)^{p_{2}} x^{p_{3}+p_{4} \ln x+p_{5}(\ln x)^{2}}
$$

where $\mathrm{x}$ is defined as:

$$
x=\frac{m_{j j}}{\sqrt{s}}
$$

with $p_{4}$ and $p_{5}$ initially set to zero. This ad-hoc fit function has been found to accurately describe the $m_{j j}$ spectrum over various center of mass energies. The number of parameters required in the background estimation function is sensitive to the luminosity collected, and an unbiased procedure

\footnotetext{
${ }^{1}$ For further information on models see Refs [2]-[3]

${ }^{2} y_{i}$ is the rapidity of the $i^{t h}$ jet, where $y=\frac{1}{2} \ln \frac{\left(E+p_{z}\right)}{\left(E-p_{z}\right)}$
} 
has been developed to test the goodness of fit using Wilks's p-value [4]. Bumphunter [5] is used to find the most discrepant region in data with respect to the background estimation (Fig 1a), taking into consideration the look-elsewhere effect [6].

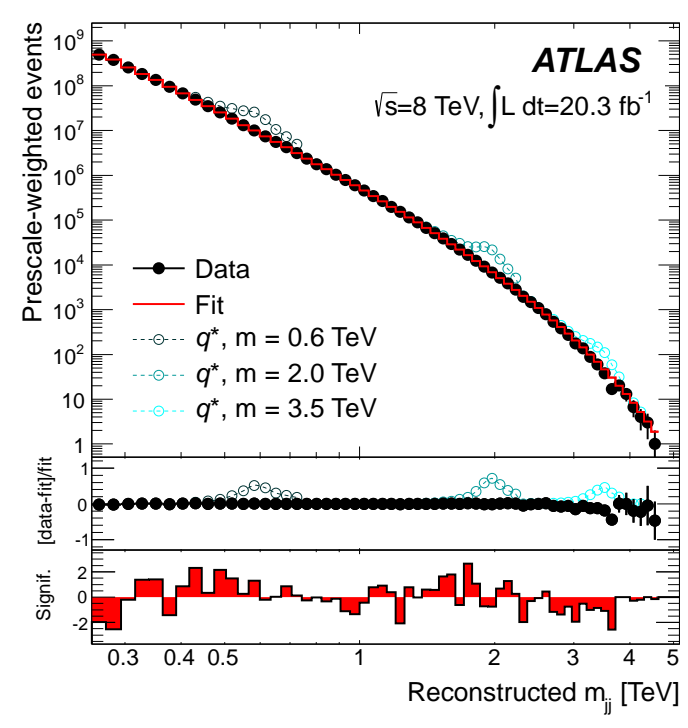

(a)

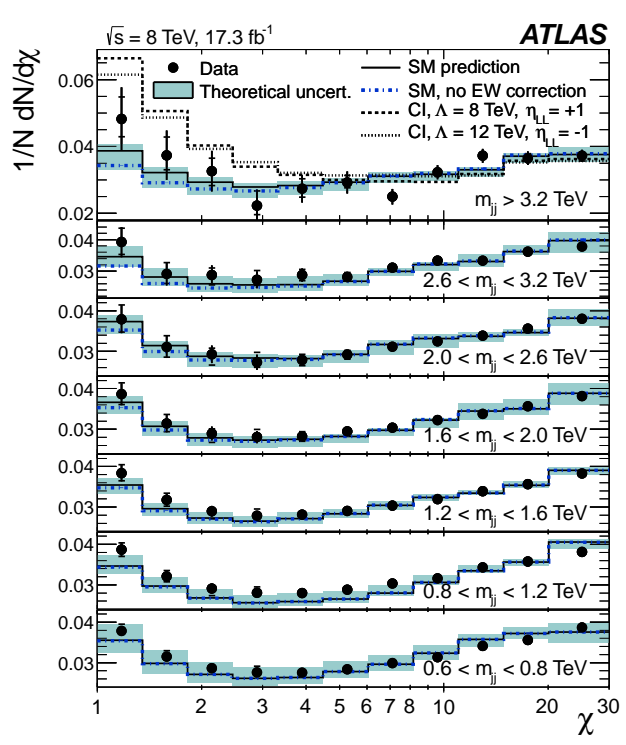

(b)

Figure 1: Dijet mass spectrum of the $8 \mathrm{TeV}$ resonance analysis (a), showing a 4 parameter background estimation and potential excited quark signals [2]. $\chi$ spectrums (b) in several $m_{j j}$ binnings for the $8 \mathrm{TeV}$ angular analysis [3]. The potential signal shape from contact interactions is shown for the $m_{j j}>3.2 \mathrm{TeV}$ bin.

QCD is expected to be fairly flat in $\chi$ while any new physics would appear as a rise at low $\chi$. The $\chi$ variable is explored in several $m_{j j}$ binnings, from low mass where new physics is already excluded to higher masses (Fig 1b). In the angular analysis the dominant QCD background shape is modeled with Pythia8 Monte Carlo corrected for NLO QCD and electroweak effects. The background normalization is set to match the data integral in each $m_{j j}$ bin.

\section{Search Potential}

Predictions of the early search potential of the resonance analysis [7] was made using 14 TeV Monte Carlo. Excited quark and QBH models were used to provide sensitivity estimates, though the analysis is model independent and limits are ultimately provided for generic gaussian resonances. Estimates have found the exclusion potential of $\mathrm{q}^{*}$ models to be competitive with Run I limits within the first few hundred $\mathrm{pb}^{-1}$ of data, and for QBH limits to be competitive well before then (Table 1). A $7 \mathrm{TeV}$ QBH signal could be discovered with only $1 \mathrm{fb}^{-1}$ of data (Fig 2a), and excluded with less Than $100 \mathrm{pb}^{-1}$ of data (Fig $2 \mathrm{~b}$ ), extending our limits well beyond the current 8 $\mathrm{TeV}$ results of $5.6 \mathrm{TeV}$.

\section{Conclusion}

ATLAS is primed to discover new physics with the start of Run 2 at the LHC. Dijet events are 


\begin{tabular}{c|c|c} 
Integrated Luminosity $\left(\mathrm{fb}^{-1}\right)$ & $m_{q^{*}}(\mathrm{TeV})$ & $m_{Q B H}(\mathrm{TeV})$ \\
\hline 0.1 & 4.0 & 8.2 \\
1 & 5.0 & 8.9 \\
5 & 5.9 & 9.2 \\
25 & 6.6 & 9.7 \\
300 & 7.4 & 10.0 \\
3000 & 8.0 & 10.1
\end{tabular}

Table 1: Expected limits on $\mathrm{q}^{*}$ and QBH models for various luminosities [7].

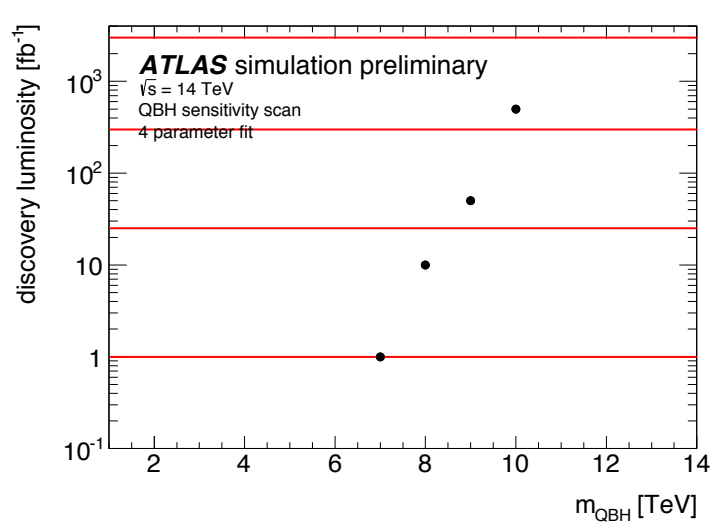

(a)

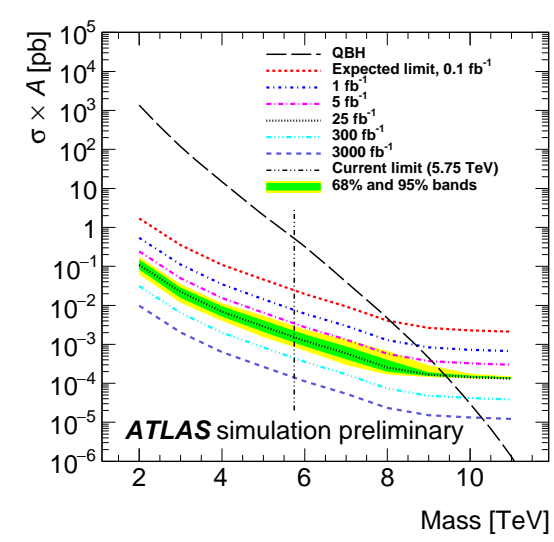

(b)

Figure 2: Luminosity discovery potential (a) and limit exclusion potential (b) for several QBH masses [7].

studied from several perspectives to provide both unique and complimentary searches and limits. Exciting results may come with the first few hundred $\mathrm{pb}^{-1}$.

\section{References}

[1] ATLAS Collaboration, The ATLAS Experiment at the CERN Large Hadron Collider, JINST 3 (2008) S08003.

[2] ATLAS Collaboration, Search for new phenomena in the dijet mass distribution using $p-p$ collision data at $\sqrt{s}=8 \mathrm{TeV}$ with the ATLAS detector, Phys. Rev. D 91, 052007 (2015) [arXiv:1407.1376].

[3] ATLAS Collaboration, Search for New Phenomena in Dijet Angular Distributions in Proton-Proton Collisions at $\sqrt{s}=8 \mathrm{TeV}$ Measured with the ATLAS Detector, Phys. Rev. Lett. 114, 221802 (2015) [arXiv:1504.00357].

[4] S.S. Wilks, The large-sample distribution of the likelihood ratio for testing composite hypotheses, Annals Math. Statist. 9, 60 (1938).

[5] G. Choudalakis, On hypothesis testing, trials factor, hypertests and the BumpHunter, [arXiv:1101.0390].

[6] E. Gross, O. Vitells, Trial factors or the look elsewhere effect in high energy physics, Eur. Phys. J. C70, 525 (2010) [arXiv:1005.1891]. 
[7] ATLAS Collaboration, Dijet resonance searches with the ATLAS detector at $14 \mathrm{TeV}$ LHC, ATL-PHYS-PUB-2015-004. 